\title{
COMPACT SUPERSYMMETRIC SOLUTIONS OF THE HETEROTIC EQUATIONS OF MOTION IN DIMENSION 5
}

\author{
MARISA FERNÁNDEZ, STEFAN IVANOV, LUIS UGARTE, AND RAQUEL VILLACAMPA
}

\begin{abstract}
We construct explicit compact supersymmetric solutions with non-zero field strength, non-flat instanton and constant dilaton to the heterotic string equations in dimension five. We present a quadratic condition on the curvature which is necessary and sufficient the heterotic supersymmetry and the anomaly cancellation to imply the heterotic equations of motion in dimension five. We supply compact nilmanifold in dimension 5 satisfying the heterotic supersymmetry equations with non-zero fluxes and constant dilaton which obey the three-form Bianchi identity and solves the heterotic equations of motion in dimension five.
\end{abstract}

\section{Contents}

1. Introduction. Field and Killing-spinor equations

2. Geometry of the heterotic supersymmetry equations

2.1. The $\mathrm{SU}(2)$-structure point of view.

3. Heterotic supersymmetry and equations of motion

3.1. Heterotic supersymmetric equations of motion with constant dilaton 10

4. Explicit compact solutions

References

\section{Introduction. Field ANd Killing-SPinOR EQUATIOns}

The bosonic fields of the ten-dimensional supergravity which arises as low energy effective theory of the heterotic string are the spacetime metric $g$, the NS three-form field strength $H$, the dilaton $\phi$ and the gauge connection $A$ with curvature $F^{A}$. The bosonic geometry considered in this paper is of the form $R^{1,9-d} \times M^{d}$ where the bosonic fields are non-trivial only on $M^{d}, d \leq 8$. We consider the two connections

$$
\nabla^{ \pm}=\nabla^{g} \pm \frac{1}{2} H
$$

where $\nabla^{g}$ is the Levi-Civita connection of the Riemannian metric $g$. Both connections preserve the metric, $\nabla^{ \pm} g=0$ and have totally skew-symmetric torsion $\pm H$, respectively.

The Green-Schwarz anomaly cancellation mechanism requires that the three-form Bianchi identity receives an $\alpha^{\prime}$ correction of the form

$$
d H=\frac{\alpha^{\prime}}{4}\left(p_{1}\left(M^{p}\right)-p_{1}(E)\right)=\frac{\alpha^{\prime}}{4} 8 \pi^{2}\left(\operatorname{Tr}(R \wedge R)-\operatorname{Tr}\left(F^{A} \wedge F^{A}\right)\right),
$$

where $p_{1}\left(M^{p}\right), p_{1}(E)$ are the first Pontrjagin forms of $M^{p}$ with respect to a connection $\nabla$ with curvature $R$ and the vector bundle $E$ with connection $A$, respectively.

A class of heterotic-string backgrounds for which the Bianchi identity of the three-form $H$ receives a correction of type (1.2) are those with $(2,0)$ world-volume supersymmetry. Such models

Date: March 1, 2019. 
were considered in 40. The target-space geometry of $(2,0)$-supersymmetric sigma models has been extensively investigated in [40, 50, 37]. Recently, there is revived interest in these models [27, 10, 28, 29, 30] as string backgrounds and in connection to heterotic-string compactifications with fluxes [9, 1, 2, 3, 45, 24, 25, 4.

In writing (1.2) there is a subtlety to the choice of connection $\nabla$ on $M^{p}$ since anomalies can be cancelled independently of the choice [38]. Different connections correspond to different regularization schemes in the two-dimensional worldsheet non-linear sigma model. Hence the background fields given for the particular choice of $\nabla$ must be related to those for a different choice by a field redefinition 49. Connections on $M^{p}$ proposed to investigate the anomaly cancellation (1.2) are $\nabla^{g}$ [50, 29], $\nabla^{+}$[10], $\nabla^{-}\left[6\right.$, 9, 30, 41], Chern connection $\nabla^{c}$ when $p=6$ [50, 45, 24, 25, 沟.

A heterotic geometry will preserve supersymmetry if and only if, in 10 dimensions, there exists at least one Majorana-Weyl spinor $\epsilon$ such that the supersymmetry variations of the fermionic fields vanish, i.e. the following Killing-spinor equations hold [50]

$$
\begin{gathered}
\delta_{\lambda}=\nabla_{m} \epsilon=\left(\nabla_{m}^{g}+\frac{1}{4} H_{m n p} \Gamma^{n p}\right) \epsilon=\nabla^{+} \epsilon=0 \\
\delta_{\Psi}=\left(\Gamma^{m} \partial_{m} \phi-\frac{1}{12} H_{m n p} \Gamma^{m n p}\right) \epsilon=\left(d \phi-\frac{1}{2} H\right) \cdot \epsilon=0 \\
\delta_{\xi}=F_{m n}^{A} \Gamma^{m n} \epsilon=F^{A} \cdot \epsilon=0,
\end{gathered}
$$

where $\lambda, \Psi, \xi$ are the gravitino, the dilatino and the gaugino fields, respectively and $\cdot$ means Clifford action of forms on spinors.

The bosonic part of the ten-dimensional supergravity action in the string frame is [6]

$$
\left.S=\frac{1}{2 k^{2}} \int d^{10} x \sqrt{-g} e^{-2 \phi}\left[S c a l^{g}+4\left(\nabla^{g} \phi\right)^{2}-\frac{1}{2}|H|^{2}-\frac{\alpha^{\prime}}{4}\left(\operatorname{Tr}\left|F^{A}\right|^{2}\right)-T r|R|^{2}\right)\right] .
$$

The string frame field equations (the equations of motion induced from the action (1.4)) of the heterotic string up to two-loops [39] in sigma model perturbation theory are (we use the notations in [30])

$$
\begin{gathered}
R i c_{i j}^{g}-\frac{1}{4} H_{i m n} H_{j}^{m n}+2 \nabla_{i}^{g} \nabla_{j}^{g} \phi-\frac{\alpha^{\prime}}{4}\left[\left(F^{A}\right)_{i m a b}\left(F^{A}\right)_{j}^{m a b}-R_{i m n q} R_{j}^{m n q}\right]=0 \\
\nabla_{i}^{g}\left(e^{-2 \phi} H_{j k}^{i}\right)=0 \\
\nabla_{i}^{+}\left(e^{-2 \phi}\left(F^{A}\right)_{j}^{i}\right)=0
\end{gathered}
$$

The field equation of the dilaton $\phi$ is implied from the first two equations above.

We search for solutions to lowest nontrivial order in $\alpha^{\prime}$ of the equations of motion that follow from the bosonic action which also preserves at least one supersymmetry.

It is known [16, 28] (30] for dimension 6), that the equations of motion of type I supergravity (1.5) with $R=0$ are automatically satisfied if one imposes, in addition to the preserving supersymmetry equations (1.3), the three-form Bianchi identity (1.2) taken with respect to a flat connection on $T M, R=0$.

A lot of effort had been done in dimension six and compact torsional solutions for the heterotic/type I string are known to exist [15, 1, 2, 10, 29, 45, 24, 25, 4, 14, 19]. In dimensions 7 and 8 non-compact heterotic/type I solutions with non-zero fluxes to the equations of motion preserving at least one supersymmetry are constructed in $[18,26,36,33$, 41] and the first compact torsional solutions are presented recently in [20].

In dimension 5, to the best of our knowledge, there are not known any compact solution either to the supersymmetry equations (1.3) or to the heterotic equations of motion (1.5) with non-zero fluxes. If the field strength vanishes, $H=0$, the 5-dimensional case reduces to dimension four 
since any five dimensional Riemannian spin manifold admitting $\nabla^{g}$-parallel spinor is reducible. Non compact solutions on circle bundle over 4-dimensional base endowed with a hyper Kähler metric when the 4-dimensional metric is Egushi-Hanson or Taub-NUT have appeared in [46], the compact cases are discussed in [29] where a cohomological obstruction is presented.

The main goal of this paper is to construct explicit compact supersymmetric valid solutions with non-zero field strength, non-flat instanton and constant dilaton to the heterotic equations of motion (1.5) in dimension 5 .

It was known [22, 23] that solutions to the first two Killing spinor equations in dimension five are quasi-Sasaki manifolds with anti-self-dual exterior derivative of the almost contact form and their special conformal transformations (see the precise definitions below). In particular, Sasakian manifolds can not solve the heterotic supersymmetry equations. In the case when the quasi-Sasaki structure is regular the solutions to the first two equations in (1.3) are $S^{1}$-bundles over a Calabi-Yau 4-manifold with anti-self-dual curvature 2 -form. Solutions we present in this paper are $S^{1}$-bundles over a 4 -torus.

In Theorem 2.5, Theorem 2.6 we give structure equations of any solution to the first two Killing spinor equations in (1.3) in terms of exterior derivatives of an $S U(2)$-structure in dimension five, a notion introduced in [12], and express its Ricci tensor in terms of the structure forms. Based on the analisys made in [22, 23] we also simplify the formula for the torsion tensor of the unique almost contact metric connection with totally skew-symmetric torsion described in [22].

According to no-go (vanishing) theorems (a consequence of the equations of motion [21, 16]; a consequence of the supersymmetry [44, 43] for $\mathrm{SU}(n)$-case and [29] for the general case) there are no compact solutions with non-zero flux and non-constant dilaton satisfying simultaneously the supersymmetry equations (1.3) and the three-form Bianchi identity (1.2) if one takes flat connection on $T M$, more precisely a connection with zero first Pontrjagin 4-form, $\operatorname{Tr}(R \wedge R)=0$. Therefore, in the compact case one necessarily has to have a non-zero term $\operatorname{Tr}(R \wedge R)$. However, under the presence of a non-zero curvature 4-form $\operatorname{Tr}(R \wedge R)$ the solution of the supersymmetry equations (1.3) and the anomaly cancellation condition (1.2) obeys the second and the third equations of motion but does not always satisfy the Einstein equation of motion (the first equation in (1.5)). We give in Theorem 3.1 a quadratic expression for $R$ which is necessary and sufficient condition in order that (1.3) and (1.2) imply (1.5) in dimension 5 based on the properties of the special geometric structure induced from the first two equations in (1.3). (A similar condition in dimension six, seven and eight we presented in [19, 20], respectively). In particular, if $R$ is an $S U(2)$-instanton the supersymmetry equations together with the anomaly cancellation condition imply the equations of motion in dimension 5 . The latter can also be seen following the considerations in the Appendix of [28].

We present in Theorem 4.4 compact nilmanifolds in dimension five satisfying the heterotic supersymmetry equations (1.3) with non-zero flux $H$, non-trivial instanton and constant dilaton obeying the three-form Bianchi identity (1.2) with curvature term $R=R^{+}$which also solve the heterotic equations of motion (1.5). Our solutions are $S^{1}$-bundles over a 4 -torus and seem to be the first explicit compact valid supersymmetric heterotic solutions with non-zero flux and constant dilaton in dimension 5 satisfying the equations of motion (1.3).

Finally, in Proposition 4.5 we show compact nilmanifolds in dimension five satisfying the heterotic supersymmetry equations (1.3) with non-zero fluxes and the three-form Bianchi identity (1.2) with curvature term $R=R^{g}$ in dimension five.

Remark 1.1. We do not know compact non-regular solutions to the first two heterotic Killing spinor equations, i.e. compact quasi-Sasaki manifolds of this kind with non-closed orbits of the Reeb vector field, or, in view of Theorem 2.5 below, compact 5-manifolds satisfying the structure equations (2.13) with non-closed orbits of the Reeb vector field. 
Our conventions: We rise and lower the indices with the metric and use the summation convention on repeated indices. For example,

$$
B_{i j k} C^{i j k}=B_{i}^{j k} C_{j k}^{i}=B_{i j k} C_{i j k}=\sum_{i j k=1}^{n} B_{i j k} C_{i j k} .
$$

The connection 1 -forms $\omega_{j i}$ of a metric connection $\nabla, \nabla g=0$ with respect to a local basis $\left\{E_{1}, \ldots, E_{n}\right\}$ are given by

$$
\omega_{j i}\left(E_{k}\right)=g\left(\nabla_{E_{k}} E_{j}, E_{i}\right),
$$

since we write $\nabla_{X} E_{j}=\omega_{j}^{s}(X) E_{s}$.

The curvature 2 -forms $\Omega_{j}^{i}$ of $\nabla$ are given in terms of the connection 1-forms $\omega_{j}^{i}$ by

$$
\Omega_{j}^{i}=d \omega_{j}^{i}+\omega_{k}^{i} \wedge \omega_{j}^{k}, \quad \Omega_{j i}=d \omega_{j i}+\omega_{k i} \wedge \omega_{j k}, \quad R_{i j k}^{l}=\Omega_{k}^{l}\left(E_{i}, E_{j}\right), \quad R_{i j k l}=R_{i j k}^{s} g_{l s} .
$$

and the first Pontrjagin class is represented by the 4-form

$$
p_{1}(\nabla)=\frac{1}{8 \pi^{2}} \sum_{1 \leq i<j \leq d} \Omega_{j}^{i} \wedge \Omega_{j}^{i} .
$$

\section{Geometry of the heterotic supersymmetry equations}

Geometrically, the vanishing of the gravitino variation is equivalent to the existence of a nontrivial real spinor parallel with respect to the metric connection $\nabla^{+}$with totally skew- symmetric torsion $T=H$. The presence of $\nabla^{+}$-parallel spinor leads to restriction of the holonomy group $\operatorname{Hol}\left(\nabla^{+}\right)$of the torsion connection $\nabla^{+}$. A detailed analysis of the induced geometries is carried out in 29] and all possible geometries (including non compact stabilizers) are investigated in [32, 34, 33, 47].

The existence of $\nabla^{+}$-parallel spinor in dimension 5 determines an almost contact metric structure whose properties as well as solutions to gravitino and dilatino Killing-spinor equations are investigated in [22, 23].

We recall that an almost contact metric structure consists of an odd dimensional manifold $M^{2 k+1}$ equipped with a Riemannian metric $g$, vector field $\xi$ of length one, its dual 1-form $\eta$ as well as an endomorphism $\psi$ of the tangent bundle such that

$$
\psi(\xi)=0, \quad \psi^{2}=-i d+\eta \otimes \xi, \quad g(\psi ., \psi .)=g(., .)-\eta \otimes \eta .
$$

The Reeb vector field $\xi$ is determined by the equations $\eta(\xi)=0, \quad \xi\lrcorner d \eta=0$, where $\lrcorner$ denotes the interior multiplication. The Nijenhuis tensor $N$ and the fundamental form $F$ of an almost contact metric structure are defined by

$$
F(., .)=g(., \psi .), \quad N=[\psi ., \psi .]+\psi^{2}[., .]-\psi[\psi ., .]-\psi[., \psi .]+d \eta \otimes \xi .
$$

There are many special types of almost contact metric structures. We introduce those which are relevant to our considerations:

- normal almost contact structures determined by the condition $N=0$;

- contact metric structures characterized by $d \eta=2 F$;

- quasi-Sasaki structures, $N=0, d F=0$. Consequently, $\xi$ is a Killing vector [0];

- Sasaki structures, $N=0, d \eta=2 F$. Consequently, $\xi$ is a Killing vector [7].

- the class of almost contact metric structures with totally skew-symmetric Nijenhuis tensor and Killing vector field $\xi$ introduced in [22]. 
In dimension five any solution to the gravitino Killing spinor equation, i.e. any parallel spinor with respect to a metric connection with torsion 3 -form defines an almost contact metric structure $(g, \xi, \eta, \psi)$ via the formulas

$$
\xi \cdot \epsilon=\sqrt{-1} \cdot \epsilon, \quad-2 \psi X \cdot \epsilon+\xi \cdot X \cdot \epsilon=\sqrt{-1} X \cdot \epsilon,
$$

which is preserved by the torsion connection.

An almost contact metric structure admits a linear connection $\nabla^{+}$with torsion 3-form preserving the structure, i.e. $\nabla^{+} g=\nabla^{+} \xi=\nabla^{+} \psi=0$, if and only if the Nijenhuis tensor is totally skew-symmetric and the vector field $\xi$ is a Killing vector field [22]. In this case the torsion connection is unique. The torsion $T$ of $\nabla^{+}$is expressed by ([22], Theorem 8.2)

$$
\left.T=\eta \wedge d \eta+d^{\psi} F+N-\eta \wedge(\xi\lrcorner N\right),
$$

where $d^{\psi} F=-d F(\psi ., \psi ., \psi)$. In particular one has $\left.\left.d \eta=\xi\right\lrcorner T, \quad \xi\right\lrcorner d \eta=0$.

In fact, (2.2) simplifies since if the Nijenhuis tensor is totally skew-symmetric then $\xi$ is a Killing vector field exactly when ([23], Proposition 3.1 and its proof)

$$
\xi\lrcorner d F=0 \Leftrightarrow \xi\lrcorner N=0 .
$$

The proof of Lemma 8.3 in 22] also yields $d \eta(.,)=.d \eta(\psi ., \psi$.$) .$

Now, Theorem 8.2 in [22] is formulated as follows

Theorem 2.1. 22, 23] An almost contact metric structure admits a unique linear connection $\nabla^{+}$with torsion 3-form preserving the structure, i.e. $\nabla^{+} g=\nabla^{+} \xi=\nabla^{+} \psi=0$, if and only if the Nijenhuis tensor is totally skew-symmetric and $\xi\lrcorner N=0$. The torsion $T$ of $\nabla^{+}$is expressed by

$$
T=\eta \wedge d \eta+d^{\psi} F+N .
$$

Since $\nabla^{+} \xi=0$ the restricted holonomy group $\operatorname{Hol}\left(\nabla^{+}\right)$of $\nabla^{+}$containes in $U(k)$. The spinor bundle $\Sigma$ of a $(2 k+1)$-dimensional almost contact metric spin manifold decomposes under the action of the fundamental 2-form $F$ into the $\operatorname{sum} \Sigma=\Sigma^{0} \oplus \cdots \oplus \Sigma^{k}, \operatorname{dim}\left(\Sigma^{r}\right)=\left(\begin{array}{l}k \\ r\end{array}\right)$. The isotropy group of a spinor of type $\Sigma^{0}$ or $\Sigma^{k}$ coincides with the subgroup $S U(k) \subset U(k)$. Consequently, there exists locally a $\nabla^{+}$-parallel spinor of type $\Sigma^{0}$ or $\Sigma^{k}$ exactly when $\operatorname{Hol}\left(\nabla^{+}\right) \subset S U(k)$. The equivalent curvature condition found in Proposition 9.1 [22] reads

$$
R_{i j k l}^{+} F^{k l}=0 \Leftrightarrow R i c_{i j}^{+}=-\nabla_{i}^{+} \theta_{j}-\frac{1}{4} \psi_{j}^{s} d T_{i s l m} F^{l m},
$$

where the Lee form $\theta$ is defined in [23] by

$$
\theta_{i}=\frac{1}{2} \psi_{i}^{s} T_{s k l} F^{k l}=\frac{1}{2} d F_{i k l} F^{k l} .
$$

Consequently, $\theta(\xi)=0$.

(Warning: note that the Lee form $\omega^{\nabla}$ defined in [22] differs slightly from $\theta, \omega^{\nabla}(\psi)=.\theta($.$) ).$

It is shown in 23] that solutions to the both gravitino and dilatino Killing spinor equations are connected with a special type 'conformal' transformations of an almost contact metric structure introduced in [23] by

$$
\psi^{\prime}:=\psi, \quad \eta^{\prime}:=\eta, \quad \xi^{\prime}:=\xi, \quad g^{\prime}:=e^{2 f} g+\left(1-e^{2 f}\right) \eta \otimes \eta,
$$

where $f$ is a smooth function which is constant along the integral curves of $\xi, d f(\xi)=0$. The new torsion $T^{\prime}$ and Lee form $\theta^{\prime}$ are given by

$$
T^{\prime}=T+\left(e^{2 f}-1\right) d^{\psi} F+2 e^{2 f} d^{\psi} f \wedge F, \quad \theta^{\prime}=\theta+2 d f,
$$

where $d^{\psi} f=-d f(\psi$.$) .$ 
We restrict our attention to dimension five. In dimension five the Nijenhuis tensor is totally skew-symmetric exactly when it vanishes [11], i.e. the structure is normal. In this case $\xi$ is automatically a Killing vector field [7], the Lee form determines completely the three form $d F$ due to (2.3), $d F=\theta \wedge F$ and the dilatino equation (the second equation in (1.3)) admits a solution exactly when the normal almost contact manifold is special conformal to a quasi-Sasaki 5-manifold ([23], Theorem 5.5). If the non-trivial spinor $\epsilon \in \Sigma^{1}$ then the space is special conformal to the standard Sasaki structure on the 5-dimensional Heisenberg group.

For a non-trivial spinor $\epsilon \in\left\{\Sigma^{0}, \Sigma^{2}\right\}$ the dilatino equation admits a solution if and only if the next equalities hold (23], Proposition 5.5)

$$
2 d \phi=\theta, \quad * \mathbb{H} d \eta=-d \eta,
$$

where $*_{\mathbb{H}}$ denote the Hodge operator acting in the 4-dimensional orthogonal complement $\mathbb{H}$ of the vector $\xi, \mathbb{H}=K e r \eta$. We call an $\mathbb{H}$-valued 2-form satisfying the second equation of (2.8) $\mathbb{H}$-anti-self dual.

In this case the torsion is given by

$$
T=\eta \wedge d \eta+2 d^{\psi} \phi \wedge F
$$

and the space is special conformal to a quasi-Sasaki 5-manifold with $\mathbb{H}$-anti-self-dual 2 form $d \eta$. In particular, there is no solution on any Sasaki 5-manifold.

Remark 2.2. The $\nabla^{+}$-parallel spinor $\epsilon \in\left\{\Sigma^{0}, \Sigma^{2}\right\}$ transforms under special conformal transformations into a $\left(\nabla^{+}\right)^{\prime}$-parallel spinor since $d \eta$ is $\mathbb{H}$-anti-self-dual [23], Theorem 5.1]. Hence, a solution to the supersymmetry equations (1.3) in dimension five reduces to solve (1.3) in the case of constant dilaton. Then any special conformal transformation gives again a solution to (1.3) and the anomaly cancellation condition could be reduced to a highly non-linear PDE for a real function $f$.

The simplest case is when the normal almost contact structure is regular, i.e. the orbit space $N^{4}=M^{5} / \xi$ is a smooth manifold. Then $M^{5}$ is a principal $S^{1}$-bundle with $\mathbb{H}$-anti-self-dual curvature form equal to $d \eta$ and any spinor $\epsilon \in\left\{\Sigma^{0}, \Sigma^{2}\right\}$ solving the gravitino and dilatino Killing spinor equation projects to $N^{4}$. Indeed, its Lie derivative $\mathbb{L}_{\xi} \epsilon$ (see [8]), calculated in [23] is

$$
\mathbb{L}_{\xi} \epsilon=\nabla_{\xi}^{g} \epsilon-\frac{1}{4} d \eta \cdot \epsilon=-\frac{1}{2} d \eta \cdot \epsilon=0
$$

since $d \eta$ is anti-self-dual and $\epsilon \in\left\{\Sigma^{0}, \Sigma^{2}\right\}$. Now, Theorem 3.2 in [23] and (2.7) yield

Theorem 2.3. 23] If $\left(M^{5}, g, \eta, \xi, \psi\right)$ is a compact regular almost contact metric manifold solving the gravitino and dilatino Killing spinor equations for $\epsilon \in\left\{\Sigma^{0}, \Sigma^{2}\right\}$ then $M^{5}$ is an $S^{1}$-bundle over a flat torus or a K3-surface with an $\mathbb{H}$-anti-self-dual curvature equal to $d \eta$. The metric has the form

$$
g_{5}=e^{2 f} g_{c y}+\eta \otimes \eta
$$

where $g_{c y}$ is the Calabi-Yau metric on the 4-dimensional base and $f$ is a smooth function on it.

The dilaton $\phi=2 f$ depends only on the Calabi-Yau 4-manifold.

The torsion is $T=\eta \wedge d \eta-2 e^{2 f}(d f \circ \psi) \wedge F$ and the flux $H=T$.

Remark 2.4. The third case of Theorem 3.2 in [23], namely $S^{1}$ bundle over the Hopf surfaces, should be excluded since this solves the dilatino equation only locally. 
2.1. The SU(2)-structure point of view. The gravitino Killing spinor equation, i.e. the $\nabla^{+}$ parallel spinor $\epsilon \in\left\{\Sigma^{0}, \Sigma^{2}\right\}$ defines a reduction of the structure group $S O(5)$ to $S U(2)$ which is described in terms of forms by Conti and Salamon in [12 as follows: an $S U(2)$-structure on 5-dimensional manifold $M$ is $\left(\eta, F=F_{1}, F_{2}, F_{3}\right)$, where $\eta$ is a 1 -form dual to $\xi$ via the metric and $F_{p}, p=1,2,3$ are 2 -forms on $M$ satisfying

$$
F_{p} \wedge F_{q}=\delta_{p q} v, \quad v \wedge \eta \neq 0
$$

for some 4-form $v$, and

$$
\left.X\lrcorner F_{1}=Y\right\lrcorner F_{2} \Rightarrow F_{3}(X, Y) \geq 0 .
$$

Now $\mathbb{H}=K e r \eta$ and the 2-forms $F_{p}, p=1,2,3$ can be chosen to form a basis of the $\mathbb{H}$-self-dual 2forms [12]. The $S U(2)$-structure $\left(\eta, F=F_{1}, F_{2}, F_{3}\right)$ is $\nabla^{+}$-parallel, $\nabla^{+} \eta=\nabla^{+} F_{p}=0, p=1,2,3$, since the defining spinor $\epsilon$ is $\nabla^{+}$-parallel.

Involving the dilatino equation, the second equation in (1.3), we get that $d \eta$ is an $\mathbb{H}$-anti-selfdual 2-form. We show that if the dilaton is constant then the 2 -forms $F_{p}, p=1,2,3$ are harmonic, i.e. closed and co-closed. We have

Theorem 2.5. The first two equations in (1.3) admit a solution with constant dilaton in dimension five exactly when there exists a five dimensional manifold $M$ endowed with an $S U(2)$-structure $\left(M, \eta, F=F_{1}, F_{2}, F_{3}\right)$ satisfying the structure equations:

$$
d F_{p}=0, \quad * \mathbb{H} d \eta=-d \eta .
$$

The Ricci tensors are given by

$$
R i c_{m n}^{+}=-d \eta_{i m} d \eta_{i n}, \quad R i c_{m n}^{g}=-\frac{1}{2} d \eta_{i m} d \eta_{i n}+\frac{1}{4}|d \eta|^{2} \eta_{m} \eta_{n}
$$

In particular, if $M$ is compact then the second and the third Betti numbers $b_{2}(M), b_{3}(M)$ are greater than or equal to three, $b_{2}(M) \geq 3, b_{3}(M) \geq 3$.

Proof. Suppose $\left(M, \eta, F=F_{1}, F_{2}, F_{3}\right)$ is a solution to the first two equations in (1.3) with constant dilaton. Then the structure is quasi-Sasaki, $N=d F_{1}=0$, the torsion of $\nabla^{+}$is given by $T=\eta \wedge d \eta$ and $d \eta$ is $\mathbb{H}$-anti-self-dual by the discussion in the previous subsection. We shall show that the $\mathbb{H}-$ self-dual forms $F_{p}$ are closed and therefore harmonic. Let $\left(e_{1}, e_{2}, e_{3}, e_{4}, e_{5}=\xi\right)$ be an orthonormal basis. Then we calculate for $X, Y, Z \in T M$ that

$$
\begin{array}{r}
\left(\nabla_{X}^{g} F_{p}\right)(Y, Z)=\left(\nabla_{X}^{+} F_{p}\right)(Y, Z)-\frac{1}{2} \sum_{s=1}^{5}\left[T\left(X, Y, e_{s}\right) F_{p}\left(e_{s}, Z\right)+T\left(X, Z, e_{s}\right) F_{p}\left(Y, e_{s}\right)\right] \\
=-\frac{1}{2} \sum_{s=1}^{4}\left[(\eta \wedge d \eta)\left(X, Y, e_{s}\right) F_{p}\left(e_{s}, Z\right)+(\eta \wedge d \eta)\left(X, Z, e_{s}\right) F_{p}\left(Y, e_{s}\right)\right]
\end{array}
$$

since $\nabla^{+} F_{p}=0$ and $\left.\xi\right\lrcorner F_{p}=0$. The equation (2.15) yields

- Let $X, Y, Z \in \mathbb{H}$. Then $\left.\nabla^{g} F_{p}\right|_{\mathbb{H}}=0$. Consequently, $\left.d F_{p}\right|_{\mathbb{H}}=0$.

- Let $X=\xi, Y, Z \in \mathbb{H}$. Then

$$
\left(\nabla_{\xi}^{g} F_{p}\right)(Y, Z)=\frac{1}{2} \sum_{s=1}^{4}\left[F_{p}\left(Z, e_{s}\right) d \eta\left(Y, e_{s}\right)-F_{p}\left(Y, e_{s}\right) d \eta\left(Z, e_{s}\right)\right]=0 .
$$

The last equality is a pure algebraic consequence of the fact that $F_{p}$ is $\mathbb{H}$-self-dual while $d \eta$ is $\mathbb{H}$-anti-self-dual. 
- Let $X, Y \in \mathbb{H}, Z=\xi$. Then

$$
\left(\nabla_{X}^{g} F_{p}\right)(Y, \xi)=\frac{1}{2} \sum_{s=1}^{4} F_{p}\left(Y, e_{s}\right) d \eta\left(X, e_{s}\right) .
$$

Using (2.16) and (2.17) we get $d F_{p}(\xi, Y, Z)=2\left(\nabla_{\xi}^{g} F_{p}\right)(Y, Z)=0$, for $Y, Z \in \mathbb{H}$. Hence, $d F_{p}=0$.

For the converse, we consider the Riemannian product $N=M \times \mathbb{R}$ with the $S U(3)$-structure $\left(\Omega, \Psi=\Psi^{+}+\sqrt{-1} \Psi^{-}\right)$with Kähler form $\Omega$ and complex volume form $\Psi$ defined by

$$
\Omega=-F_{1}-\eta \wedge d t ; \quad \Psi^{+}=F_{2} \wedge \eta-F_{3} \wedge d t ; \quad \Psi^{-}=F_{3} \wedge \eta+F_{2} \wedge d t .
$$

Using (2.13) we easily derive from (2.18) that $d(\Omega \wedge \Omega)=d \Psi^{+}=d \Psi^{-}=0$, i.e. it is a balanced hermitian structure with holomorphic complex volume form. In particular, the almost contact metric structure on $M$ is normal. Applying Theorem 4.1 and Corollary 4.3 from [41] we conclude

$$
\nabla_{N}^{+} \Omega=\nabla_{N}^{+} \Psi^{+}=\nabla_{N}^{+} \Psi^{-}=0,
$$

where $\nabla_{N}^{+}$is the Bismut-Strominger metric connection with torsion 3-form $T_{N}$ given by

$$
T_{N}=-*_{N} d \Omega=\eta \wedge d \eta
$$

where $*_{N}$ denotes the Hodge operator on $N$ and we have used the first equation in (2.18) and the fact that $d \eta$ is $\mathbb{H}$-anti-self-dual.

Hence, the torsion $T_{N}$ does not depend on $\mathbb{R}$ and therefore the connection $\nabla_{N}^{+}$descends to $M$. Now, (2.19) yield $\nabla_{N}^{+} \eta=\nabla_{N}^{+} F_{p}=0, p=1,2,3$ and the descended connection on $M$ coincides with $\nabla^{+}$as two metric connections with equal torsion must coincide.

The formulas for the Ricci tensors (2.14) follow just taking $d f=0$ into 2.23) established below.

The last assertion follows from the fact that the three 2 -forms $F_{p}$ and the three 3 -forms $F_{p} \wedge \eta$ are harmonic and represent different cohomology classes. Indeed, the equation $*_{\mathbb{H}} F_{p}=F_{p}$ implies $\delta F_{p}=-* d * F_{p}=-* d\left(F_{p} \wedge \eta\right)=-*\left(d F_{p} \wedge \eta+F_{p} \wedge d \eta\right)=0$ since $d \eta$ is $\mathbb{H}$-anti-self-dual.

Combine Remark 2.2 with Theorem 2.5 using (2.7) to derive

Theorem 2.6. The first two equations in (1.3) admit a solution in dimension five exactly when there exists a five dimensional manifold $M$ endowed with an $S U(2)$-structure $(M, \eta, F=$ $\left.F_{1}, F_{2}, F_{3}\right)$ satisfying the structure equations:

$$
d F_{p}=2 d f \wedge F_{p}, \quad *_{\mathbb{H}} d \eta=-d \eta, \quad d f(\xi)=0 .
$$

The flux $H$ is given by

$$
H=T=\eta \wedge d \eta+2 d^{\psi} f \wedge F,
$$

where $\psi$ is the almost complex structure on $\mathbb{H}$ defined by $g(X, \psi Y)=F(X, Y)$

The dilaton $\phi$ is equal to $\phi=2 f$.

The Ricci tensors are given by:

$$
\begin{gathered}
\operatorname{Ric}_{m n}^{+}=-d \eta_{i m} d \eta_{i n}+\nabla_{m}^{g} d f_{n}+\Delta_{\mathbb{H}} f g_{m n}+d f_{i}\left(d \eta_{i m} \eta_{n}-d \eta_{i n} \eta_{m}\right) \\
R i c_{m n}^{g}=-\frac{1}{2} d \eta_{i m} d \eta_{i n}+\frac{1}{4}|d \eta|^{2} \eta_{m} \eta_{n}+\nabla_{m}^{g} d f_{n}+\Delta_{\mathbb{H}} f g_{m n}-2 d f_{m} d f_{n}+2|d f|^{2} g_{m n}
\end{gathered}
$$

where $\Delta_{\mathbb{H}} f:=\sum_{i=1}^{4}\left(\nabla_{e_{i}}^{g} d f\right) e_{i}$ is the horizontal subLaplacian and $|d f|^{2}:=\sum_{i=1}^{4} d f\left(e_{i}\right)^{2}$ is the norm of the horizontal gradient.

In particular, if $M$ is compact then the second and the third Betti numbers $b_{2}(M), b_{3}(M)$ are greater than or equal to three, $b_{2}(M) \geq 3, b_{3}(M) \geq 3$. 
Proof. We need to prove only (2.23). We shall use (3.4) and (3.3) from below. Using (2.22) and (2.21), we calculate

$$
\begin{gathered}
-\frac{1}{4} \psi_{n}^{s} d T_{m s i j} F^{i j}=-4 d \eta_{i m} d \eta_{i n}+2\left(d d^{\psi} f\right)_{i j} F^{i j}-8|d f|^{2} g_{m n} \\
2\left(d d^{\psi} f\right)_{i j} F^{i j}=-4 \nabla_{i}^{+} d f_{i}+2 d f_{i} \psi_{i}^{j} T_{j p q} F^{p q}=-4 \Delta_{\mathbb{H}} f+8|d f|^{2} \\
d f_{i} T_{i m n}=d f_{i}\left(d \eta_{i m} \eta_{n}-d \eta_{i n} \eta_{m}\right) ; \\
T_{m i j} T_{n i j}=2 d \eta_{m i} d \eta_{n i}+|d \eta|^{2} \eta_{m} \eta_{n}-8 d f_{m} d f_{n}+8|d f|^{2} g_{m n} .
\end{gathered}
$$

We get the first equality in (2.23) from (3.4) and the first three equalities in (2.24). The second equality follows from the already proved first one, the fourth equality in (2.24) and (3.3) below.

We note that another proof of the second equality in $(2.14),(2.23)$ can be derived from the general formula of the Ricci tensor for a general $S U(2)$-structure presented in [5].

In addition to gravitino and dilatino Killing spinor equations, the vanishing of the gaugino variation requires the 2 -form $F^{A}$ to be of instanton type ( 13, 50, 36, 48, 17, 29]). In dimension five, an $S U(2)$-instanton i.e the gauge field $A$ is a connection with curvature 2 -form $F^{A} \in s u(2)$. The $S U(2)$-instanton condition can be written in the form [13, 50]

$$
F_{m n}^{A}=-\frac{1}{2} F_{p q}^{A}(F \wedge F)^{p q}{ }_{m n} .
$$

In this paper we consider compact regular $S U(2)$ manifolds in dimension 5 , more precisely the case of $S^{1}$-bundles over a flat 4 -torus. We find a compact solution to (1.3) satisfying the anomaly cancellation (1.2) with non-zero fluxes, constant dilaton which also solves the heterotic equations of motion (1.5).

Remark 2.7. It is interesting whether there are compact non-regular (the integral curves of the Reeb vector fielf $\xi$ are not closed) quasi-Sasaki 5-manifolds with anti-self-dual 2-form d $\eta$ whose Riemannian Ricci tensor is given by (2.14), or equivalently, non-regular SU(2)- structures obeying (2.13) on compact 5-manifold. We do not know any examples of this kind. Such examples might be relevant in constructing compact heterotic solutions in dimension six since the construction of $\mathbb{T}^{2}$-bundles over Calabi-Yau surface presented in [31] can be generalized to a circle bundle over such an example solving automatically the first two equations in (1.3) (see [20]).

\section{Heterotic Supersymmetry AND EQUations of motion}

It is known [16, 28] (30] for dimension 6), that the equations of motion of type I supergravity (1.5) with $R=0$ are automatically satisfied if one imposes, in addition to the preserving supersymmetry equations (1.3), the three-form Bianchi identity (1.2) taken with respect to a flat connection on $T M, R=0$. However, the no-go theorems [21, 16, 44, 43, 29] state that if even $\operatorname{Tr} R \wedge R=0$ there are no compact solutions with non-zero flux $H$ and non-constant dilaton.

In the presence of a curvature term $\operatorname{Tr} R \wedge R \neq 0$ a solution of the supersymmetry equations (1.3) and the anomaly cancellation condition (1.2) obeys the second and the third equations in (1.5) but does not always satisfy the Einstein equation of motion (the first equation in (1.5)). However if the curvature $R$ is of instanton type (1.3) and (1.2) imply (1.5) which can also be seen follow the considerations in the Appendix of [28]. We have

Theorem 3.1. The Einstein equation of motion (the first equation in (1.5)) is a consequence of the heterotic Killing spinor equations (1.3) and the anomaly cancellation (1.2) if and only if the next identity holds

$$
\frac{1}{2}\left[R_{m s a b} R_{p q a b}+R_{m p a b} R_{q s a b}+R_{m q a b} R_{s p a b}\right] F^{p q} \psi_{n}^{s}=R_{m p q r} R_{n}^{p q r} .
$$


In particular, if $R$ is an instanton then (3.1) holds.

Proof. The Ricci tensors are connected by (see e.g. [22])

$$
\begin{gathered}
R i c_{m n}^{g}=R i c_{m n}^{+}+\frac{1}{4} T_{m p q} T_{n}^{p q}-\frac{1}{2} \nabla_{s}^{+} T_{m n}^{s}, \quad R i c_{m n}^{+}-R i c_{n m}^{+}=\nabla_{s}^{+} T_{m n}^{s}=\nabla_{s}^{g} T_{m n}^{s}, \\
R i c_{m n}^{g}=\frac{1}{2}\left(R i c_{m n}^{+}+R i c_{n m}^{+}\right)+\frac{1}{4} T_{m p q} T_{n}^{p q} .
\end{gathered}
$$

In view of (2.8), the Ricci tensor described in (2.5) is given by

$$
R i c_{m n}^{+}=-2 \nabla_{m}^{+} d \phi_{n}-\frac{1}{4} \psi_{n}^{s} d T_{m s i j} F^{i j}=-2 \nabla_{m}^{g} d \phi_{n}+d \phi_{s} T_{m n}^{s}-\frac{1}{4} \psi_{n}^{s} d T_{m s i j} F^{i j}
$$

Substitute (3.4) into (3.3), insert the result into the first equation of (1.5) and use the anomaly cancellation (1.2) to conclude the assertion.

It is shown in [42] that the curvature of $R^{+}$satisfies the identity $R_{i j k l}^{+}=R_{k l i j}^{+}$if and only if $\nabla_{i}^{+} T_{j k l}$ is a four form. Now Theorem 3.1 yields

Corollary 3.2. Suppose the torsion 3-form is $\nabla^{+}$-parallel, $\nabla_{i}^{+} T_{j k l}=0$. The equations of motion (1.5) with respect to the curvature $R^{+}$of the $(+)$-connection are consequences of the heterotic Killing spinor equations (1.3) and the anomaly cancellation (1.2).

3.1. Heterotic supersymmetric equations of motion with constant dilaton. In the case when the dilaton is constant we arrive to the following problems:

We look for a compact 5-manifold $M$ with an $S U(2)$-structure $\left(\eta, F_{p}\right), p=1,2,3$ which satisfies the following conditions

a). Gravitino and dilatino Killing spinor equations (the first two equations in (1.3): the forms $F_{p}$ are closed and $d \eta$ is $\mathbb{H}$-anti-self-dual.

b). Gaugino Killing spinor equation (the third equation in (1.3)). Look for a vector bundle $E$ of rank $r$ over $M$ equipped with an $S U(2)$-instanton, i.e. a connection $A$ with curvature 2 -form $\Omega^{A}$ satisfying

$$
\left(\Omega^{A}\right)_{E_{i}, E_{j}}\left(\psi E_{k}, \psi E_{l}\right)=\left(\Omega^{A}\right)_{E_{i}, E_{j}}\left(E_{k}, E_{l}\right), \quad \sum_{k=1}^{5}\left(\Omega^{A}\right)_{E_{i}, E_{j}}\left(E_{k}, \psi E_{k}\right)=0,
$$

where $\left\{E_{1}, \ldots, E_{5}=\xi\right\}$ is an orthonormal basis on $M$ and $\psi$ is the almost complex structure on $\mathbb{H}$ defined by $g(X, \psi Y)=F_{1}(X, Y)$.

c). Anomaly cancellation condition:

$$
d H=d T=d \eta \wedge d \eta=\frac{\alpha^{\prime}}{4} 8 \pi^{2}\left(p_{1}(M)-p_{1}(A)\right), \quad \alpha^{\prime}>0 .
$$

d). The first Pontrjagin form $p_{1}(M)$ satisfies equation (3.1).

\section{EXPLiCIT COMPACT SOLUTIONS}

In this section we give an explicit family of compact solutions to the heterotic supersymmetric equations of motion with constant dilaton, based on a quotient of the 5-dimensional generalized Heisenberg group $H(2,1)$. 
First, let us recall that $H(2,1)$ is the nilpotent Lie group consisting of the matrices of the form

$$
H(2,1)=\left\{\left(\begin{array}{cccc}
1 & x_{1} & x_{2} & z \\
0 & 1 & 0 & y_{1} \\
0 & 0 & 1 & y_{2} \\
0 & 0 & 0 & 1
\end{array}\right) \mid x_{i}, y_{i}, z \in \mathbb{R}, 1 \leq i \leq 2\right\}
$$

For each triple $(a, b, c) \in \mathbb{R}^{3}$ such that $a^{2}+b^{2} \neq 0$, we consider the basis of left invariant 1-forms $e^{1}, \ldots, e^{5}$ on $H(2,1)$ given by

$$
\begin{array}{ll}
e^{1}=\left(a^{2}+b^{2}+c^{2}\right) d x_{1}, & e^{2}=a d y_{1}+b\left(1+\frac{c^{2}}{a^{2}+b^{2}}\right) d x_{2}-a c d y_{2}, \\
e^{3}=b d y_{1}-a\left(1+\frac{c^{2}}{a^{2}+b^{2}}\right) d x_{2}-b c d y_{2}, & e^{4}=c d y_{1}+\left(a^{2}+b^{2}\right) d y_{2}, \\
e^{5}=\left(a^{2}+b^{2}+c^{2}\right)^{2}\left(x_{1} d y_{1}+x_{2} d y_{2}-d z\right) . &
\end{array}
$$

In terms of this basis the structure equations of the Lie algebra $\mathfrak{h}(2,1)$ of $H(2,1)$ become

$$
\left\{\begin{array}{l}
d e^{1}=d e^{2}=d e^{3}=d e^{4}=0, \\
d e^{5}=a\left(e^{12}-e^{34}\right)+b\left(e^{13}+e^{24}\right)+c\left(e^{14}-e^{23}\right) .
\end{array}\right.
$$

Notice that these equations also correspond to $\mathfrak{h}(2,1)$ when $a=b=0$ and $c \neq 0$, so (4.1) are valid for any triple $(a, b, c) \in \mathbb{R}^{3}-\{(0,0,0)\}$. It is immediate to check that the $S U(2)$-structure $\left(\eta, F_{1}, F_{2}, F_{3}\right)$ given by

$$
F_{1}=e^{12}+e^{34}, \quad F_{2}=e^{13}+e^{42}, \quad F_{3}=e^{14}+e^{23}, \quad \eta=e^{5} .
$$

satisfies (2.13). In view of Theorem 2.5, this family provides explicit solutions (with constant dilaton) to the first two equations in (1.3).

Next we prove that this is the unique family of left invariant solutions (with constant dilaton) to the first two equations in (1.3) on a 5-dimensional Lie group.

Theorem 4.1. Let $\mathfrak{g}$ be a Lie algebra of dimension 5 with an $S U(2)$-structure $\left(\eta, F_{1}, F_{2}, F_{3}\right)$ satisfying

$$
d F_{1}=0, \quad d F_{2}=0, \quad d F_{3}=0, \quad *_{\mathbb{H}} d \eta=-d \eta \neq 0 .
$$

Then, $\mathfrak{g}$ is isomorphic to the Lie algebra $\mathfrak{h}(2,1)$. Moreover, there is a basis $e^{1}, \ldots, e^{5}$ for $\mathfrak{g}^{*}$ satisfying (4.1) for some $a, b, c \in \mathbb{R}$ with $a^{2}+b^{2}+c^{2} \neq 0$ and such that the $S U(2)$-structure $\left(\eta, F_{1}, F_{2}, F_{3}\right)$ expresses as $(4.2)$.

Proof. Let us consider a basis $e^{1}, \ldots, e^{5}$ for $\mathfrak{g}^{*}$ such that the $S U(2)$-structure $\left(\eta, F_{1}, F_{2}, F_{3}\right)$ expresses as (4.2). In terms of $e^{1}, \ldots, e^{5}$ the equations of the Lie algebra $\mathfrak{g}$ are of the form

$$
\left\{\begin{aligned}
d e^{i}= & a_{i 1} F_{1}+a_{i 2} F_{2}+a_{i 3} F_{3}+b_{i 1} F_{1}^{-}+b_{i 2} F_{2}^{-}+b_{i 3} F_{3}^{-} \\
& +\left(c_{i 1} e^{1}+c_{i 2} e^{2}+c_{i 3} e^{3}+c_{i 4} e^{4}\right) e^{5} \\
d e^{5}= & a\left(e^{12}-e^{34}\right)+b\left(e^{13}+e^{24}\right)+c\left(e^{14}-e^{23}\right)
\end{aligned}\right.
$$

for $i=1, \ldots, 4$, where $a_{i j}, b_{i j}, c_{i j} \in \mathbb{R}$ and

$$
F_{1}^{-}=e^{12}-e^{34}, \quad F_{2}^{-}=e^{13}+e^{24}, \quad F_{3}^{-}=e^{14}-e^{23} .
$$

Let us denote by $F_{i}^{j k l}$ the component in $e^{j k l}$ of the 3 -form $d F_{i}$. It is easy to see that

$$
F_{1}^{125}=-c_{11}-c_{22}, \quad F_{2}^{135}=-c_{11}-c_{33}, \quad F_{3}^{145}=-c_{11}-c_{44}, \quad F_{1}^{345}=-c_{33}-c_{44},
$$


which imply the vanishing of $c_{i i}$, for $i=1, \ldots, 4$. Moreover,

$$
\begin{array}{llll}
F_{1}^{135}=-c_{23}+c_{41}, & F_{1}^{245}=c_{14}-c_{32}, & F_{2}^{125}=-c_{32}-c_{41}, & F_{2}^{345}=c_{14}+c_{23}, \\
F_{1}^{145}=-c_{24}-c_{31}, & F_{1}^{235}=c_{13}+c_{42}, & F_{3}^{125}=c_{31}-c_{42}, & F_{3}^{345}=-c_{13}+c_{24}, \\
F_{2}^{145}=c_{21}-c_{34}, & F_{2}^{235}=-c_{12}+c_{43}, & F_{3}^{135}=-c_{21}-c_{43}, & F_{3}^{245}=-c_{12}-c_{34},
\end{array}
$$

which imply the following equalities:

$$
c_{41}=c_{23}=-c_{32}=-c_{14}, \quad c_{42}=c_{31}=-c_{24}=-c_{13}, \quad c_{43}=-c_{34}=-c_{21}=c_{12} .
$$

Let $E_{1}, \ldots, E_{5}$ be the basis of $\mathfrak{g}$ dual to $e^{1}, \ldots, e^{5}$, and let us denote by $P_{i j k}^{l}$ the component in $E_{l}$ of $\left[\left[E_{i}, E_{j}\right], E_{k}\right]+\left[\left[E_{j}, E_{k}\right], E_{i}\right]+\left[\left[E_{k}, E_{i}\right], E_{j}\right]$, i.e.

$$
\left[\left[E_{i}, E_{j}\right], E_{k}\right]+\left[\left[E_{j}, E_{k}\right], E_{i}\right]+\left[\left[E_{k}, E_{i}\right], E_{j}\right]=\sum_{l=1}^{5} P_{i j k}^{l} E_{l}
$$

It is clear that the Jacobi identity of the Lie algebra $\mathfrak{g}$ is equivalent to $P_{i j k}^{l}=0$ for $1 \leq i<j<$ $k \leq 5$ and $1 \leq l \leq 5$. From the vanishing of $c_{i i}$ and (4.4), a direct calculation shows that

$$
P_{235}^{5}=-2\left(b c_{12}-a c_{13}\right), \quad P_{245}^{5}=-2\left(c c_{12}-a c_{14}\right), \quad P_{345}^{5}=-2\left(c c_{13}-b c_{14}\right) .
$$

Therefore, $P_{235}^{5} P_{245}^{5}=P_{345}^{5}=0$ if and only if there is $\lambda \in \mathbb{R}$ such that

$$
c_{12}=\lambda a, \quad c_{13}=\lambda b, \quad c_{14}=\lambda c .
$$

Moreover,

$$
\begin{aligned}
& \begin{cases}P_{125}^{1}+P_{345}^{1}=2 \lambda\left(a a_{21}+b a_{31}+c a_{41}\right), & P_{125}^{2}+P_{345}^{2}=-2 \lambda\left(a a_{11}+c a_{31}-b a_{41}\right), \\
P_{125}^{3}+P_{345}^{3}=-2 \lambda\left(b a_{11}-c a_{21}+c a a_{41}\right), & P_{125}^{4}+P_{345}^{4}=-2 \lambda\left(c a_{11}+b a_{21}-a a_{31}\right),\end{cases} \\
& \begin{cases}P_{135}^{1}-P_{245}^{1}=2 \lambda\left(a a_{22}+b a_{32}+c a_{42}\right), & P_{135}^{2}-P_{245}^{2}=-2 \lambda\left(a a_{12}+c a_{32}-b a_{42}\right), \\
P_{135}^{3}-P_{245}^{3}=-2 \lambda\left(b a_{12}-c a_{22}+a a_{42}\right), & P_{135}^{4}-P_{245}^{4}=-2 \lambda\left(c a_{12}+b a_{22}-a a_{32}\right),\end{cases} \\
& \begin{cases}P_{145}^{1}+P_{235}^{1}=2 \lambda\left(a a_{23}+b a_{33}+c a_{43}\right), & P_{145}^{2}+P_{235}^{2}=-2 \lambda\left(a a_{13}+c a_{33}-b a_{43}\right), \\
P_{145}^{3}+P_{235}^{3}=-2 \lambda\left(b a_{13}-c a_{23}+a a_{43}\right), & P_{145}^{4}+P_{235}^{4}=-2 \lambda\left(c a_{13}+b a_{23}-a a_{33}\right),\end{cases}
\end{aligned}
$$

If $\lambda \neq 0$, since $a^{2}+b^{2}+c^{2} \neq 0$, then it follows from (4.6), (4.7) and (4.8) that $a_{11}=a_{21}=$ $a_{31}=a_{41}=0, a_{12}=a_{22}=a_{32}=a_{42}=0$ and $a_{13}=a_{23}=a_{33}=a_{43}=0$, respectively. Now, $\mathrm{a}$ direct calculation similar to the given above shows that $P_{235}^{l}=P_{245}^{l}=P_{345}^{l}=0$ for $l=1, \ldots, 4$ if and only if all the coefficients $b_{i j}$ are zero. But then $P_{124}^{3}=\lambda\left(a^{2}+b^{2}+c^{2}\right) \neq 0$, that is to say, the Jacobi identity is not satisfied. This proves that the coefficient $\lambda$ in (4.5) vanishes.

Since $\lambda=0$ then (4.4) and (4.5) imply that all the coeffcients $c_{i j}$ in (4.3) vanish, and therefore the Lie algebra $\mathfrak{g}$ is an extension of a 4-dimensional Lie algebra $\mathfrak{a}$ having a triple of 2 -forms $F_{1}, F_{2}, F_{3}$ satisfying $F_{i} \wedge F_{i}=F_{j} \wedge F_{j} \neq 0$ and $F_{i} \wedge F_{j}=0$ for $i \neq j$. This gives a hyperKähler structure on $\mathfrak{a}$ and it follows that the Lie algebra $\mathfrak{a}$ is necessarily abelian. Therefore, all the coefficients $a_{i j}, b_{i j}, c_{i j}$ vanish, i.e. (4.3) reduces to (4.1) and $\mathfrak{g}$ is isomorphic to $\mathfrak{h}(2,1)$.

From now on, we restrict our attention to the family of $S U(2)$-structures $\left(\eta, F_{1}, F_{2}, F_{3}\right)$ given by (4.1)-4.2). Let $\Gamma(2,1)$ denote the subgroup of matrices of $H(2,1)$ with integer entries and consider the compact nilmanifold $N(2,1)=\Gamma(2,1) \backslash H(2,1)$. We can describe $N(2,1)$ as a principal circle bundle over a 4 -torus

$$
S^{1} \hookrightarrow N(2,1) \rightarrow T^{4},
$$


by the projection $\left(x_{1}, y_{1}, x_{2}, y_{2}, z\right) \mapsto\left(x_{1}, y_{1}, x_{2}, y_{2}\right)$. Since the $\mathrm{SU}(2)$-structure given by (4.1)(4.2) is left invariant, it descend to an $\mathrm{SU}(2)$-structure on the compact manifold $N(2,1)$ satisfying (2.13).

Since the torsion 3-form $T$ of the $S U(2)$-structure is

$$
T=\eta \wedge d \eta=a e^{125}+b e^{135}+c e^{145}-c e^{235}+b e^{245}-a e^{345},
$$

we have that

$$
d T=-2\left(a^{2}+b^{2}+c^{2}\right) e^{1234}
$$

It is straightforward to check that $T$ is parallel with respect to the torsion connection $\nabla^{+}$, i.e.

Lemma 4.2. For any $a, b, c \in \mathbb{R}$ such that $a^{2}+b^{2}+c^{2} \neq 0$, we have $\nabla^{+} T=0$.

On the other hand, using (1.1) and the expression (4.9), we calculate the non-zero curvature forms $\left(\Omega^{+}\right)_{j}^{i}=-\left(\Omega^{+}\right)_{i}^{j}$ of the torsion connection are determined by:

$$
\left(\Omega^{+}\right)_{2}^{1}=-\left(\Omega^{+}\right)_{4}^{3}=-a d e^{5}, \quad\left(\Omega^{+}\right)_{3}^{1}=\left(\Omega^{+}\right)_{4}^{2}=-b d e^{5}, \quad\left(\Omega^{+}\right)_{4}^{1}=-\left(\Omega^{+}\right)_{3}^{2}-c d e^{5} .
$$

Next we find a large family of $\mathrm{SU}(2)$-instantons.

Proposition 4.3. Let $A_{\lambda, \mu, \tau}$ be the linear connection on $N(2,1)$ defined by the connection forms

$$
\begin{aligned}
& \left(\sigma^{A_{\lambda, \mu, \tau}}\right)_{2}^{1}=-\left(\sigma^{A_{\lambda, \mu, \tau}}\right)_{1}^{2}=-\left(\sigma^{A_{\lambda, \mu, \tau}}\right)_{4}^{3}=\left(\sigma^{A_{\lambda, \mu, \tau}}\right)_{3}^{4}=-\lambda e^{5}, \\
& \left(\sigma^{A_{\lambda, \mu, \tau}}\right)_{3}^{1}=-\left(\sigma^{A_{\lambda, \mu, \tau}}\right)_{1}^{3}=\left(\sigma^{A_{\lambda, \mu, \tau}}\right)_{4}^{2}=-\left(\sigma^{A_{\lambda, \mu, \tau}}\right)_{2}^{4}=-\mu e^{5}, \\
& \left(\sigma^{A_{\lambda, \mu, \tau}}\right)_{4}^{1}=-\left(\sigma^{A_{\lambda, \mu, \tau}}\right)_{1}^{4}=-\left(\sigma^{A_{\lambda, \mu, \tau}}\right)_{3}^{2}=\left(\sigma^{A_{\lambda, \mu, \tau}}\right)_{2}^{3}=-\tau e^{5},
\end{aligned}
$$

and $\left(\sigma^{A_{\lambda, \mu, \tau}}\right)_{j}^{i}=0$ for the remaining $(i, j)$, where $\lambda, \mu, \tau \in \mathbb{R}$. Then, $A_{\lambda, \mu, \tau}$ is an $S U(2)$-instanton with respect to any of the $S U(2)$-structures $\left(\eta, F_{1}, F_{2}, F_{3}\right)$ given by (4.1)-(4.2), $A_{\lambda, \mu, \tau}$ preserves the metric, and its first Pontrjagin form is given by

$$
p_{1}\left(A_{\lambda, \mu, \tau}\right)=-\frac{\left(\lambda^{2}+\mu^{2}+\tau^{2}\right)\left(a^{2}+b^{2}+c^{2}\right)}{2 \pi^{2}} e^{1234} .
$$

Proof. A direct calculation shows that the non-zero curvature forms $\left(\Omega^{A_{\lambda, \mu, \tau}}\right)_{j}^{i}$ of the connection $A_{\lambda, \mu, \tau}$ are:

$$
\begin{aligned}
& \left(\Omega^{A_{\lambda, \mu, \tau}}\right)_{2}^{1}=-\left(\Omega^{A_{\lambda, \mu, \tau}}\right)_{1}^{2}=-\left(\Omega^{A_{\lambda, \mu, \tau}}\right)_{4}^{3}=\left(\Omega^{A_{\lambda, \mu, \tau}}\right)_{3}^{4}=-\lambda d e^{5}, \\
& \left(\Omega^{A_{\lambda, \mu, \tau}}\right)_{3}^{1}=-\left(\Omega^{A_{\lambda, \mu, \tau}}\right)_{1}^{3}=\left(\Omega^{A_{\lambda, \mu, \tau}}\right)_{4}^{2}=-\left(\Omega^{A_{\lambda, \mu, \tau}}\right)_{2}^{4}=-\mu d e^{5} \\
& \left(\Omega^{A_{\lambda, \mu, \tau}}\right)_{4}^{1}=-\left(\Omega^{A_{\lambda, \mu, \tau}}\right)_{1}^{4}=-\left(\Omega^{A_{\lambda, \mu, \tau}}\right)_{3}^{2}=\left(\Omega^{A_{\lambda, \mu, \tau}}\right)_{2}^{3}=-\tau d e^{5} .
\end{aligned}
$$

Hence $A_{\lambda, \mu, \tau}$ satisfies (3.5); in fact, since $F_{1}=e^{12}+e^{34}$, the almost complex structure $\psi$ is given by $\psi\left(E_{1}\right)=-E_{2}, \psi\left(E_{3}\right)=-E_{4}$. Therefore, the connection $A_{\lambda, \mu, \tau}$ is an $S U(2)$-instanton.

The following results give explicit compact valid solutions on $N(2,1)$ to the heterotic supersymmetry equations with non-zero flux and constant dilaton satisfying the anomaly cancellation condition with respect to $\nabla^{+}$and the Levi-Civita connection $\nabla^{g}$ and show that all our solutions for $\nabla^{+}$also solve the equations of motion.

Theorem 4.4. Let $N(2,1)$ be a compact $S U(2)$-nilmanifold as above, $\nabla^{+}$the torsion connection and $A_{\lambda, \mu, \tau}$ the $S U(2)$-instanton given in Proposition 4.3 . Let $(\lambda, \mu, \tau) \neq(0,0,0)$ be such that $\lambda^{2}+\mu^{2}+\tau^{2}<a^{2}+b^{2}+c^{2} ;$ then

$$
d T=2 \pi^{2} \alpha^{\prime}\left(p_{1}\left(\nabla^{+}\right)-p_{1}\left(A_{\lambda, \mu, \tau}\right)\right),
$$

where $\alpha^{\prime}=2\left(a^{2}+b^{2}+c^{2}-\lambda^{2}-\mu^{2}-\tau^{2}\right)^{-1}>0$. 
Therefore, the nilmanifold manifold $\left(N(2,1), \eta, F_{s}, A_{\lambda, \mu, \tau}, \nabla^{+}\right)$is a compact solution to the supersymmetry equations (1.3) obeying the anomaly cancellation (1.2) and solving the equations of motion (1.5) in dimension 5.

Denote $r=a^{2}+b^{2}+c^{2}$, the Riemannian metric can be expressed locally by

i) If $(a, b) \neq(0,0)$ then

$$
g=r^{2}\left(d x_{1}\right)^{2}+r^{2}\left(d x_{2}\right)^{2}+r\left(d y_{1}\right)^{2}+r\left(a^{2}+b^{2}\right)\left(d y_{2}\right)^{2}+r^{4}\left(x_{1} d y_{1}+x_{2} d y_{2}-d z\right)^{2},
$$

ii) If $a=b=0$ then

$$
g=\left(d x_{1}\right)^{2}+\left(d x_{2}\right)^{2}+\left(d y_{1}\right)^{2}+\left(d y_{2}\right)^{2}+c^{2}\left(x_{1} d y_{1}+x_{2} d y_{2}-d z\right)^{2} .
$$

Proof. The non-zero curvature forms of the torsion connection $\nabla^{+}$are given by (4.11), which implies that its first Pontrjagin form is

$$
p_{1}\left(\nabla^{+}\right)=-\frac{\left(a^{2}+b^{2}+c^{2}\right)^{2}}{2 \pi^{2}} e^{1234} .
$$

Now the proof follows directly from $(4.10)$ and Proposition 4.3. The final assertion in the theorem follows from Lemma 4.2 and Corollary 3.2 .

Proposition 4.5. Let $N(2,1)$ be a compact $S U(2)$-nilmanifold as above, $\nabla^{g}$ the Levi-Civita connection and $A_{\lambda, \mu, \tau}$ the $S U(2)$-instanton given in Proposition 4.⿹. Let $(\lambda, \mu, \tau) \neq(0,0,0)$ be such that $\lambda^{2}+\mu^{2}+\tau^{2}<\frac{3}{8}\left(a^{2}+b^{2}+c^{2}\right) ;$ then

$$
d T=2 \pi^{2} \alpha^{\prime}\left(p_{1}\left(\nabla^{g}\right)-p_{1}\left(A_{\lambda, \mu, \tau}\right)\right),
$$

where $\alpha^{\prime}=16\left(3\left(a^{2}+b^{2}+c^{2}\right)-8\left(\lambda^{2}+\mu^{2}+\tau^{2}\right)\right)^{-1}>0$.

Therefore, the nilmanifold manifold $\left(N(2,1), \eta, F_{s}, A_{\lambda, \mu, \tau}, \nabla^{g}\right)$ is a compact solution to the supersymmetry equations (1.3) satisfying the anomaly cancellation condition (1.2).

Proof. The non-zero curvature forms $\left(\Omega^{g}\right)_{j}^{i}=-\left(\Omega^{g}\right)_{i}^{j}$ of the Levi-Civita connection $\nabla^{g}$ are determined by $\left(\Omega^{g}\right)_{5}^{i}=\frac{1}{4}\left(a^{2}+b^{2}+c^{2}\right) e^{i 5}$, for $i=1, \ldots, 4$, and

$$
\begin{array}{ll}
\left(\Omega^{g}\right)_{2}^{1}=-\frac{3 a}{4} d e^{5}-\frac{1}{4}\left(a^{2}+b^{2}+c^{2}\right) e^{34}, & \left(\Omega^{g}\right)_{3}^{1}=-\frac{3 b}{4} d e^{5}+\frac{1}{4}\left(a^{2}+b^{2}+c^{2}\right) e^{24}, \\
\left(\Omega^{g}\right)_{4}^{1}=-\frac{3 c}{4} d e^{5}-\frac{1}{4}\left(a^{2}+b^{2}+c^{2}\right) e^{23}, & \left(\Omega^{g}\right)_{3}^{2}=\frac{3 c}{4} d e^{5}-\frac{1}{4}\left(a^{2}+b^{2}+c^{2}\right) e^{14} \\
\left(\Omega^{g}\right)_{4}^{2}=-\frac{3 b}{4} d e^{5}+\frac{1}{4}\left(a^{2}+b^{2}+c^{2}\right) e^{13}, & \left(\Omega^{g}\right)_{4}^{3}=\frac{3 a}{4} d e^{5}-\frac{1}{4}\left(a^{2}+b^{2}+c^{2}\right) e^{12} .
\end{array}
$$

This implies that the first Pontrjagin form of $\nabla^{g}$ is

$$
p_{1}\left(\nabla^{g}\right)=-\frac{3}{16 \pi^{2}}\left(a^{2}+b^{2}+c^{2}\right)^{2} e^{1234} .
$$

Now the proof follows directly from (4.10) and Proposition 4.3.

Remark 4.6. The first Pontrjagin form of the connection $\nabla^{-}$is zero, therefore there is no compact solution to the heterotic supersymmetry equations satisfying the anomaly cancellation condition with $\nabla=\nabla^{-}$.

Acknowledgments. This work has been partially supported through grant MEC (Spain) MTM200508757-C04-02 and under project "Ingenio Mathematica (i-MATH)" No. CSD2006-00032 (Consolider Ingenio 2010). S.I. is partially supported by the Contract $154 / 2008$ with the University of Sofia 'St.Kl.Ohridski'. S.I. is a Senior Associate to the Abdus Salam ICTP, Trieste and the final stage of the research was done during his stay in the ICTP, Fall 2008. 


\section{REFERENCES}

[1] K. Becker, M. Becker, K. Dasgupta, P.S. Green, Compactifications of Heterotic Theory on Non-Kähler Complex Manifolds: I, JHEP 0304 (2003) 007.

[2] K. Becker, M. Becker, K. Dasgupta, P.S. Green, E. Sharpe, Compactifications of Heterotic Strings on NonKähler Complex Manifolds: II, Nucl. Phys. B678 (2004), 19-100.

[3] K. Becker, M. Becker, K. Dasgupta, S. Prokushkin, Properties from heterotic vacua from superpotentials, hep-th/0304001.

[4] K. Becker, M. Becker, J-X. Fu, L-S. Tseng, S-T. Yau, Anomaly Cancellation and Smooth Non-Kahler Solutions in Heterotic String Theory, Nucl.Phys. B751 (2006) 108-128.

[5] L. Bedulli, L. Vezzoni, Torsion of SU(2)-structures and Ricci curvature in dimension 5, arXiv:math/0702790.

[6] E. A. Bergshoeff, M. de Roo, The quartic effective action of the heterotic string and supersymmetry, Nucl. Phys. B 328 (1989), 439.

[7] D. Blair, Contact manifolds in Riemannian geometry, Lect. Notes Math. 509, Springer-Verlag, 1976.

[8] J.P. Bourguignon, P. Gauduchon, Spineurs, operateurs de Dirac et variations de metriques, Comm. Math. Phys. 144 (1992), 581-599.

[9] G. L. Cardoso, G. Curio, G. Dall'Agata, D. Lust, BPS Action and Superpotential for Heterotic String Compactifications with Fluxes, JHEP 0310 (2003) 004.

[10] G.L. Cardoso, G. Curio, G. Dall'Agata, D. Lust, P. Manousselis, G. Zoupanos, Non-Käeler string back-grounds and their five torsion classes, Nuclear Phys. B 652 (2003), 5-34.

[11] D. China, J.C. Marrero, Classifications of almost contact metric structures, Rev. Roumaine Math. Pures Appl. 37 (1992), 581-599.

[12] D. Conti, S. Salamon, Generalized Killing spinors in dimension 5, Trans. Amer. Math. Soc. 359 (2007), 53195343.

[13] E. Corrigan, C. Devchand, D.B. Fairlie, J. Nuyts, First-order equations for gauge fields in spaces of dimension greater than four, Nuclear Phys. B 214 (1983), no. 3, 452-464.

[14] K. Dasgupta, H. Firouzjahi, R. Gwyn, On the warped heterotic axion, arXiv:0803.3828 [hep-th], to appear in JHEP.

[15] K. Dasgupta, G. Rajesh, S. Sethi, M theory, orientifolds and G-flux, JHEP 0211, 006 (2002).

[16] B. de Wit, D.J. Smit, N.D. Hari Dass, Residual Supersimmetry Of Compactified D=10 Supergravity, Nucl. Phys. B 283 (1987), 165.

[17] S.K. Donaldson, R.P. Thomas, Gauge theory in higher dimensions, The geometric universe (Oxford, 1996), 31-47, Oxford Univ. Press, Oxford, 1998.

[18] D.B. Fairlie, J. Nuyts, Spherically symmetric solutions of gauge theories in eight dimensions, J. Phys. A17 (1984) 2867.

[19] M.Fernandez, S.Ivanov, L.Ugarte, R.Villacampa, Non-Kaehler heterotic-string compactifications with non-zero fluxes and constant dilaton, Comm. Math. Phys., to appear, arXiv:0804.1648.

[20] M.Fernandez, S.Ivanov, L.Ugarte, R.Villacampa, Compact supersymmetric solutions of the heterotic equations of motion in dimensions 7 and 8, arXiv:0806.4356.

[21] D.Z. Freedman, G.W. Gibbons, P.C. West, Ten Into Four Won't Go, Phys. Lett. B 124 (1983), 491.

[22] Th. Friedrich, S. Ivanov Parallel spinors and connections with skew-symmetric torsion in string theory, Asian J. Math. 6 (2002), 3003-336.

[23] Th. Friedrich, S. Ivanov, Almost contact manifolds, connections with torsion, parallel spinors, J. reine angew. Math. 559 (2003), 217-236.

[24] J-X. Fu, S-T. Yau, Existence of Supersymmetric Hermitian Metrics with Torsion on Non-Kaehler Manifolds, arXiv:hep-th/0509028.

[25] J-X. Fu, S-T. Yau, The theory of superstring with flux on non-Kähler manifolds and the complex Monge-Ampere equation, arXiv:hep-th/0604063.

[26] S. Fubini, H. Nikolai, The octonionic instanton, Phys. Let. B 155 (1985) 369.

[27] J. Gauntlett, N. Kim, D. Martelli, D. Waldram, Fivebranes wrapped on SLAG three-cycles and related geometry, JHEP 0111 (2001) 018.

[28] J.P. Gauntlett, D. Martelli, S. Pakis, D. Waldram, G-Structures and Wrapped NS5-Branes, Commun. Math. Phys. 247 (2004), 421-445.

[29] J. Gauntlett, D. Martelli, D. Waldram, Superstrings with Intrinsic torsion, Phys. Rev. D69 (2004) 086002.

[30] J. Gillard, G. Papadopoulos, D. Tsimpis, Anomaly, Fluxes and (2,0) Heterotic-String Compactifications, JHEP 0306 (2003) 035. 
[31] E. Goldstein, S. Prokushkin, Geometric Model for Complex Non-Käehler Manifolds with SU(3) Structure, Commun. Math. Phys. 251 (2004), 65-78.

[32] U. Gran, P. Lohrmann, G. Papadopoulos, The spinorial geometry of supersymmetric heterotic string backgrounds, JHEP 0602 (2006) 063.

[33] U. Gran, G. Papadopoulos, D. Roest Supersymmetric heterotic string backgrounds, Phys. Lett. B 656 (2007), 119.

[34] U. Gran, G. Papadopoulos, D. Roest, P. Sloane Geometry of all supersymmetric type I backgrounds, JHEP 0708 (2007) 074.

[35] M. Günaydin, H. Nikolai, Seven-dimensional octonionic Yang-Mills instanton and its extension to an heterotic string soliton, Phys. Lett. B 353 (1991) 169.

[36] J.A. Harvey, A. Strominger, Octonionic superstring solitons, Phys. Review Let. 665 (1991) 549.

[37] P.S. Howe, G. Papadopoulos, Ultraviolet behavior of two-dimensional supersymmetric non-linear sigma models, Nucl. Phys. B 289 (1987), 264.

[38] C.M. Hull, Anomalies, ambiquities and superstrings, Phys. lett. B167 (1986), 51.

[39] C.M. Hull, P.K. Townsend, The two loop beta function for sigma models with torsion, Phys. Lett. B 191 (1987), 115.

[40] C.M. Hull, E. Witten, Supersymmetric sigma models and the Heterotic String, Phys. Lett. B 160 (1985), 398.

[41] P. Ivanov, S. Ivanov, SU(3)-instantons and $G_{2}$, Spin(7)-Heterotic string solitons, Comm. Math. Phys. 259 (2005), 79-102.

[42] S. Ivanov, Geometry of quaternionic Kähler connections with torsion, J. Geom. Phys. 41 (2002), no. 3, $235-257$.

[43] S. Ivanov, G. Papadopoulos, A no-go theorem for string warped compactifications, Phys.Lett. B497 (2001) 309-316.

[44] S. Ivanov, G. Papadopoulos, Vanishing Theorems and String Backgrounds, Class.Quant.Grav. 18 (2001) 10891110 .

[45] J. Li, S-T. Yau, The Existence of Supersymmetric String Theory with Torsion, J. Diff. Geom. 70, no. 1, (2005).

[46] H. Lu, J.F. Vazquez-Poritz, Resolution of overlaping branes, Phys. Lett. B 534 (2002), 155.

[47] G. Papadopoulos, Solution of heterotic Killing spinor equations and special geometry, arXiv:0811.1539 [math.DG].

[48] R. Reyes Carrión, A generalization of the notion of instanton, Diff. Geom. Appl. 8 (1998), no. 1, 1-20.

[49] A. Sen, (2,0) supersymmetry and space-time supersymmetry in the heterotic strin theory, Nucl. Phys. B 167 (1986), 289.

[50] A. Strominger, Superstrings with torsion, Nucl. Phys. B 274 (1986) 253.

(Fernández) Universidad del País Vasco, Facultad de Ciencia y Tecnología, Departamento de Matemáticas, Apartado 644, 48080 Bilbao, Spain

E-mail address: marisa.fernandez@ehu.es

(Ivanov) University of Sofia "St. Kl. Ohridski", Faculty of Mathematics and Informatics, Blvd. JAMEs Bourchier 5, 1164 Sofia, Bulgaria

E-mail address: ivanovsp@fmi.uni-sofia.bg

(Ugarte) Departamento de Matemáticas-I.U.M.A., Universidad de Zaragoza, Campus Plaza San Francisco, 50009 Zaragoza, Spain

E-mail address: ugarte@unizar.es

(Villacampa) Departamento de Matemáticas - I.U.M.A., Universidad de Zaragoza, Campus Plaza San Francisco, 50009 Zaragoza, Spain

E-mail address: raquelvg@unizar.es 